\title{
Treatment of Severe Recalcitrant Fungal Keratitis Using Subconjunctival Fluconazole as an Adjunctive Therapy
}

\author{
Ambreen Gul ${ }^{1}$, Fuad Ahmad Khan Niazi ${ }^{2}$, Ali Raza ${ }^{3}$ \\ ${ }^{1-3}$ Department of Ophthalmology, Holy Family Hospital, Rawalpindi Medical University, Rawalpindi
}

\begin{abstract}
Purpose: To evaluate the safety and effectiveness of $2 \%$ subconjunctival injection of Fluconazole as an adjunctive treatment in severe recalcitrant fungal keratitis.
\end{abstract}

Study Design: Interventional case series.

Place and Duration of Study: Ophthalmology department of Holy family hospital, Rawalpindi medical university, from January 2019 to August 2019.

Methods: The study included 18 eyes of 18 patients with severe resistant fungal corneal ulcer.We excluded those cases who had known hypersensitivities to Fluconazole. These ulcers were refractory toprimary conventional antifungal therapy with topical Natamycin, topical and systemic Fluconazole. Sample for culture was taken with sterile cotton bud and scraping was taken with kimura spatula. All resistant cases were given1.0 ml of $2 \%$ subconjunctival Fluconazole injection once a day for at least one week. After that depending upon the condition, the injections were givenonalternate days for 2 weeks.

Results: Average age of the patients was 35.22 years (SD \pm 10.42$)$. Among total 18 patients, six (33.33\%) were females and $12(66.67 \%)$ were males. Thirteen (72.22\%) cases showed absolute response after one week and 5 $(27.77 \%)$ cases needed more injections. Four $(22.22 \%)$ of these 5 cases partiallyimproved andone case failed to show improvement. Final vision varied in different cases according to the position of the residual scar. Five cases ended up in keratoplasty. Local or systemic toxicity was not seen in any case.

Conclusion: Subconjunctival injection of $2 \%$ Fluconazole can be used as an adjunctive therapy for severe fungal keratitis without any toxic complications.

Key Words: Keratomycosis, Evisceration, Keratoplasty.

How to Cite this Article: Gul A, Niazi FAK, Raza A. Treatment of Severe Recalcitrant Fungal Keratitis Using Subconjunctival Fluconazole as an Adjunctive Therapy. Pak J Ophthalmol. 2021, 37 (2): 173-178.

Doi: http://doi.org/10.36351/pjo.v37i2.1081

\section{INTRODUCTION}

Globally, one of the common reasons of unilateral

Correspondence: Ambreen Gul

Department of Ophthalmology, Holy Family Hospital, Rawalpindi Medical University, Rawalpindi

Email:amber-gul@hotmail.com

Received: June 13, 2020

Accepted: March 2, 2021 blindness is keratitis. In majority of the cases, corneal infections are treatable or avoidable. ${ }^{1}$ In developing countries like Pakistan, the second most common cause of visual impairment is central corneal opacity, cataract being the first. ${ }^{2}$ Keratitis can be secondary to infectious agent or trivial trauma to eye. Infectious keratitis, a sight threatening inflammation and necrosis of stromal layer of cornea, is commonly correlated with micro pathogens like bacteria, virus or fungus. ${ }^{3}$ Microbial keratitis leads to irreversible loss of vision due tosequel like suppuration, corneal melting, 
vascularization and opacification of cornea. ${ }^{4}$

Of all the infectious keratitis pathogens, fungi have been reported to be the most virulent and intangible, posing a diagnostic and therapeutic challenge to ophthalmic clinicians worldwide, particularly in tropical countries with temperate warm zones. ${ }^{5}$ Mycotic keratitis is accounted to be roughly $44 \%$ among all other causes of keratitis in developing countries. ${ }^{6}$ Precipitating factors leading to increased incidence includeinjury to eye especially vegetative, contact lenses usage, immunosuppression caused by steroids and antibiotics usage and systemic illnesses like diabetes and agricultural occupation. ${ }^{7}$ These pathogens invade the defective epithelium leading to penetration of corneal stroma causing immunologic inflammation and destruction leading to devastating tissue injury and scarring. ${ }^{8}$ Keratitis which is refractory to medical treatment leads to progressive disease like perforated corneal ulcers at presentation. In $15-27 \%$ of fungal infections the outcome is surgical intervention like keratoplasty, evisceration or enucleation. ${ }^{9}$ Majority of therapeutic keratoplasties are performed for severe, advanced and non-healing ulcers. ${ }^{9}$

Numerous types of fungi such as yeast like Candida Albicans, dimorphic and filamentous pathogens like Fusarium and Aspergillus are responsible for fungal keratitis. Etiologyof fungal keratitis and prevalence ofparticularmicrobes in specific geographic areas demonstrates inconsistency. Other robust pathogens include Mucor and Alternaria. ${ }^{10}$ Early detection of fungal keratitis and referral along with justified medical treatment are imperative for favorable prognosis. Reasons for medical treatment failure include difficulty in availability of antifungal agents, limited penetration of the drugs, cost and toxic side effects. Despite advances in medical treatment, cure of fungal keratitis is complex. One of the reasons is that majority of the antifungal drugs are fungi static and not fungicidal. ${ }^{11}$ Treatment modalities based on routesof administering drugs are direct topical, intra stromal, intracameral, subconjunctival, intravitreal and systemic antifungal agents. There are various therapeutic drug regimens but all groups have their own benefits and side effects. ${ }^{12}$ Commonly used drugs are polyenes such as amphotericin, Natamycin and azoles like Fluconazole, itraconazole. Choice of agent and route depends on severity and type of fungal infection. First line of treatment is with topical polyenes. If keratitis is deep with full thickness stromal or endothelial involvement and aqueous or anterior chamber invasion, subconjunctival, intra-cameral or intra-stromal routes are preferred. Intravitreal injections are reserved for keratitis related endophthalmitis. Systemic antifungals are used for scleritis or endophthalmitis but their efficacy is not clear. ${ }^{13}$ Advanced drugs like azoles are superior to polyenes in better penetration,broad antifungal spectrum, less toxicity and are relatively cheaper. Fluconazole is the first generation Bistriazole with added benefit of less protein binding, slow metabolism and fewer side effects. Due to better ocular penetration, it achieves higher levels in anterior chamber for longer duration. ${ }^{14,15}$ Sub-conjunctival injection of highly toxic drugs like Amphotericin and Natamycin is discouraged due to thinning of sclera and necrosis of conjunctiva. ${ }^{16}$ Sub-conjunctival injection of $2 \%$ Fluconazole has been shown to offer pharmacokinetic benefits as well as broad spectrum coverage. ${ }^{17}$

The rationale of the present study is to scrutinize and report the effectiveness and safety of subconjunctival injection of $2 \%$ Fluconazole in treating severe recalcitrant fungal keratitis, refractory to conservative antifungal treatment regimens.

\section{METHODS}

After taking approval from the institutional review board, current study enrolled eighteen patients with clinical diagnosis of severe fungal keratitis. The patients were of the grade 2 or 3 Jones criteria (mentioned in table 1) and not responding to or deteriorating on conservative topical and systemic antifungal medication. We excluded those cases who had known hypersensitivities to Fluconazole. Informed written consent was obtained regarding the drug injection and its reported pros and cons. After detailed history and demographic data entry, we examined the patients in cornea clinic and slit lamp biomicroscopy was done. Corneal culturewas taken with sterile cotton bud and scraping was taken with kimura spatula and put on sterile glass slide. The samples were sent to microbiology laboratory. Microscopic inspection of hyphae or pseudohyphae was set as a diagnostic criteriafor fungal keratitis. Hyphae were considered as molds and pseudohyphae as yeast by microscopic analysis. In those patients, who had both negative stain and culture, aqueous tap was done and sent for culture sensitivity. All patients were admitted in hospital and 
advised first-line therapy of Natamycin eye drops four hourly, topical Fluconazole 2\% eye drops one hourly, Atropine $1 \%$ eye drops twice a day and Moxifloxacin eye drops to prevent secondary bacterial keratitis. If the ulcer worsened and showed no response to conventional regimen, oral Fluconazole was added (200 mg) on day 1 then $100 \mathrm{mg}$ daily for two weeks after assessing liver function tests.In patients who failed to respond after 14 days, sub-conjunctival Fluconazole injection was started. The intravenous solution of Fluconazole 2\%, up to $1.0 \mathrm{ml}$, was injected sub-conjunctivaly in infero-temporal fornix once daily for at least 7 days. All injections were given by single consultant ophthalmologist under topical anesthesia. After the first week, those cases who still had hypopyon or stromal infiltration, sub-conjunctival injections were continued on alternate days for 2 weeks. The patients were followed up for six months after discharge to observe recurrent infection. Patients were divided into three groups on the basis of response to treatment.

\section{Group A}

Cured patientswho showed absolute response to the treatment with complete resolution of corneal stromal/endothelial infiltrates and hypopyon following $2 \%$ sub-conjunctival Fluconazole injection for 7 days.

\section{Group B}

Incomplete response was defined as partial resolution of primary infection including infiltrates and hypopyon after 7 days of $2 \%$ sub-conjunctival Fluconazole injection.

\section{Group C}

Noresponse was defined as not responding primary infectionandadvanced endophthalmitis leading to surgical intervention.

Table 1: Jones grading criteria.

\begin{tabular}{|c|c|c|c|}
\hline Factor & Grade 1 & Grade 2 & Grade 3 \\
\hline Location & Non axial & $\begin{array}{l}\text { Central or } \\
\text { peripheral }\end{array}$ & $\begin{array}{l}\text { Central or } \\
\text { peripheral }\end{array}$ \\
\hline Area & $2 \mathrm{~mm}$ & $2-6 \mathrm{~mm}$ & $>6 \mathrm{~mm}$ \\
\hline Depth & $\begin{array}{l}\text { Superficial } \\
\text { one third }\end{array}$ & $\begin{array}{l}\text { Superficial } \\
\text { two third }\end{array}$ & $\begin{array}{l}\text { Extending to } \\
\text { inner one third }\end{array}$ \\
\hline $\begin{array}{l}\text { Anterior Segment } \\
\text { Inflammation }\end{array}$ & Mild & Moderate & $\begin{array}{l}\text { Severe hypopyon/ } \\
\text { fibrionus exudate }\end{array}$ \\
\hline
\end{tabular}

\section{RESULTS}

Average age of the patientswas 35.22 years (SD \pm 10.42 ) with a range of 16 to 60 years. Among 18 patients, six $(33.33 \%)$ were women and $12(66.67 \%)$ were men.Culture sensitivity results are shown in Figure 1.Four (22.22\%) patients had diabetes mellitus; two $(11.11 \%)$ had tuberculosis, two $(11.11 \%)$ had rheumatoid arthritis, rest of the patients had no systemic risk factors. Eleven eyes (61.11\%) had history of vegetative trauma along with organic foreign body. Four eyes (22.22\%) had history of longterm use of topical corticosteroid or corticosteroidantibiotic combination therapy. Eight (44.44\%) patients were agriculturists and fieldworkers. Peripheral corneal ulcer was present in $13(72.22 \%)$ patients while $5(27.77 \%)$ patients had central keratitis. Size of the infiltrate was $3 \mathrm{~mm}$ in $3(16.66 \%)$ cases,3$6 \mathrm{~mm}$ in $11(61.11 \%)$ cases and $>6 \mathrm{~mm}$ in $4(22.22 \%)$ cases. Depth of infiltration was superficial $2 / 3^{\text {rd }}$ in 13 $(72.22 \%)$ cases and extending to inner $1 / 3^{\text {rd }}$ leading to endothelial plaque in $5(27.77 \%)$ cases. $4(22.22 \%)$ cases had corneal thinning with impending perforation and 1 case had descemetocele at presentation.

Total of 17 cases showed effective response. Thirteen were cured with complete resolution of infection and fourpatients partially responded requiring repeated injections. One patient of our series showed no response and underwent evisceration. In 13 cases sub-conjunctival injection of Fluconazole 2\% $1 \mathrm{ml}$ was given for 7 days. Five patients required repeated injections. Hypopyon resolved after repeated sub-conjunctival Fluconazole therapy within mean 6.23 days. One patient with descemetocele did not show any signs of improvement in primary infection

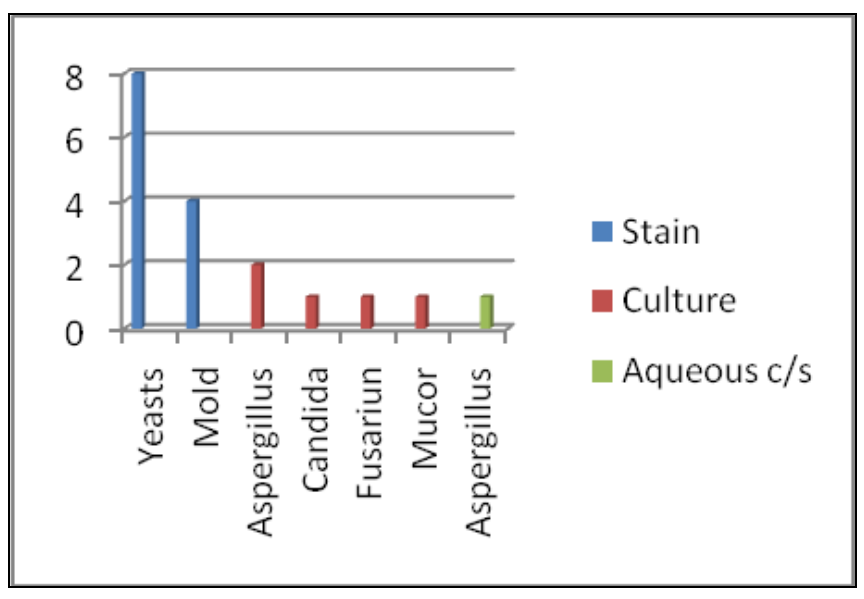

Figure 1: Culture Sensitivity and Staining Results. 
or hypopyon. Ulcer healed within 4.8 weeks. Four cases ultimately required penetrating keratoplasty for severe corneal scarring and it was done 6 months after ulcer resolution. Three patients presented with recurrent fungal keratitis within 4 weeks of last subconjunctival Fluconazole. They were managed with repeated Subconjunctival Fluconazole injections and they showed partial response. Only ocular side effect reported was sub-conjunctival hemorrhage. Patients were kept on follow-up for at least 6 months for side effects or recurrence.

Pre-treatment and post-treatment visual acuity is shown in table 2.

Table 2: Pre-Treatment and Post Treatment Visual Acuity.

\begin{tabular}{lclc}
\hline $\begin{array}{l}\text { Presenting Visual } \\
\text { Acuity }\end{array}$ & $\begin{array}{c}\text { No of } \\
\text { Cases }\end{array}$ & $\begin{array}{l}\text { Post Treatment Visual } \\
\text { Acuity }\end{array}$ & $\begin{array}{c}\text { No of } \\
\text { cases }\end{array}$ \\
\hline Perception of Light & 3 & No Perception of Light & 1 \\
Hand Movements & 9 & Hand movements & 1 \\
Counting Fingers & 6 & Counting finger-6/60 & 3 \\
6/60-6/24 & - & 6/36-6/24 & 9 \\
6/18-6/12 & - & $6 / 18-6 / 12$ & 4 \\
\hline
\end{tabular}

\section{DISCUSSION}

Prevalence of fungal keratitis is high in tropical and subtropical developing countries leading to severe ocular morbidity. It is one of the principle causes of blindness with strikingly poorer prognosis than other infectious keratitis. Majority of the cases are associated with vegetative traumain field workers from rural areas and delayed presentation often results in advanced complicated cases. ${ }^{1,5}$ In Pakistan, the reported incidence of fungal keratitis cases was $38.45 \%$ according to a study conducted in a tertiary care centre in Larkana. ${ }^{18}$ This reported incidence is comparable to 44.1\% amongst Mettapracharak patients and also identical to that reported China. ${ }^{19}$

On account of narrow spectrum, medical therapy failure often leads to call for early surgical intervention like penetrating keratolpasty and enucleation/evisceration. In a study carried out by Xie $\mathrm{L}$ et al, $35.1 \%$ of fungal keratitis patients received keratoplasty treatment for not responding to medical therapy. Although therapeutic PKP is effective in curing and preserving the eye along with visual rehabilitation but it is also associated with complications like recurrent fungal keratitis, graft rejection and failure requiring intensive antifungal therapy. ${ }^{8,9}$ Availability of good grafts is a limitation in our setups. It is said that one cornea is available for 70 patients who require corneal graft, leading to severe imbalance between global supply and demand. Majority of the patients with advanced complicated fungal keratitis end up in evisceration before getting access to corneal transplant. ${ }^{20}$

For deep fungal keratitis with hypopyon, higher concentrations of drug isrequired in the anterior chamber for longer duration and that can be achievedwith sub-conjunctival injections. Problem with sub-conjunctival administration of antifungal agents is toxicity especially polyenes. Azoles group of antifungal drugs have enhancedocular penetration against several fungal pathogens. ${ }^{21}$ Fluconazole $2 \%$ solution is used as topical agent as well. In comparison to Amphotericin B it has been recognized to have very low ocular toxicity and superior ocular penetration. Intact epithelium leads to lesser penetration of antifungal agents but epithelial debridement in already thin corneas can lead to progression or worsening of ulcer along with risk of perforation. Fluconazole has been reported to show equal penetration in debrided and non-debrided corneas of fungal keratitis. ${ }^{22}$

Role of sub-conjunctival Fluconazole has been reported in few clinical studies of fungal keratitis not responding to conventional antifungal agents. However, there are no clear guidelines for dosage and frequency of administration. Yilmaz et al conducted a study on role of $2 \%$ Subconjunctival Fluconazole 1 mltwice a day for 5 days initiallyand then once a day for 2 weeksin 13 patients and they found that 12 patients healed and one patient required enucleation. ${ }^{23}$ Sachin Dev et al reported a study of 33 patients who received $0.5 \mathrm{ml}$ of $0.2 \%$ Subconjunctival Fluconazole once a day. Out of total 33 patients, 18 responded to treatment. They reported that subconjunctival injection was safer for a maximum of 60 days. ${ }^{24}$ Isipradit $S$ et al reported 50 percent cure rate and $83.3 \%$ successful treatment in a study of 6 patients who received $0.5 \mathrm{ml}$ of $0.2 \%$ Subconjunctival Fluconazole twice a day for initial 5 days then once a day for 14 days along with intracameral Amphotericin B and topical antifungal and oral Itraconazole. ${ }^{25}$

In the current study, signs of clinical improvement as hypopyon resolution or decrease in size of infiltrate was seenas early as $3^{\text {rd }}$ day after first injection in eyes with Candidainfection. In eyes with Fusarium, Mucor and Aspergillus, response was seen as late as 12 days after first subconjunctival injection. Yilmaz et al reported healing after 10 injections and seven patients required $5-14$ days of additional injections. ${ }^{23}$ 
Limitations of our study are small sample size and negative cultures in many cases. It could be due to scrapping done superficially because of corneal thinning and melting, but inner $1 / 3^{\text {rd }}$ of stroma was also involved in many cases. It could also be due to the fact that patients were already taking antifungal before referral to our hospital.

\section{CONCLUSION}

Prompt and timely diagnosis of fungal keratitis along with combined fortified antifungal agents and subconjunctival Fluconazole 2\% for grade 2 and 3 fungal keratitis is a good strategy for non-responding ulcers.

\section{Ethical Approval}

The study was approved by the Institutional review board/ Ethical review board. (86/IREF/RMU/2019).

\section{Conflict of Interest}

Authors declared no conflict of interest.

\section{REFERENCES}

1. Acharya Y, Acharya B, Karki P. Fungal keratitis: study of increasing trend and common determinants. Nepal J Epidemiol 2017; 7 (2): 685-693.

2. Dineen B, Bourne RA, Jadoon Z. Causes of blindness and visual impairment in Pakistan. The Pakistan national blindness and visual impairment survey. $\mathrm{Br} \mathbf{J}$ Ophthalmol. 2007; 91 (8): 1005-1010.

3. Srigyan D, Gupta M, Behera HS. Keratitis: An inflammation of cornea. EC Ophthalmol. 2017; 6 (6): 171-177.

4. Austin A, Lietman T, Rose-Nussbaumer J. Update on the management of infectious keratitis. Ophthalmology, 2017; 124 (11): 1678-1689.

5. Manikandan $\mathbf{P}$, Abdel-hadi A, Singh YRB. Fungal keratitis: Epidemiology, Rapid detection, and Antifungal susceptibilities of Fusarium and Aspergillus Isolates from corneal scrapings. Bio Med Res Intern. 2019: 6395840.

6. Saha S, Banerjee D, Khetan A. Epidemiological profile of fungal keratitis in urban population of West Bengal, India. Oman J Ophthalmol. 2009; 2 (3): 114118.

7. Narsani AK, Gul S, Dabir SA. Fungal keratitis: 84 cases report in Southern Pakistan. Int J Ophthalmol. 2009; 2 (2): 154-157.

8. Niu L, Liu X, Ma Z, Yin Y, Sun L, Yang L, et al. Fungal keratitis: Pathogenesis, diagnosis and prevention. Microb Pathog. 2020; 138: 103802.
9. Xie L, Dong X, Shi W. Treatment of fungal keratitis by penetrating keratoplasty. Br J Ophthalmol. 2001; 85: 1070-1074.

10. Tanure MA, Cohen EJ, Sudesh S, Rapuano CJ, Laibson PR. Spectrum of fungal keratitis at Wills Eye Hospital, Philadelphia, Pennsylvania. Cornea, 2000; 19: 307-312.

11. Ansari Z, Miller D, Galor A. Current thoughts in fungal keratitis: Diagnosis and treatment. Curr Fungal Infect Rep. 2013; 7 (3): 209-218.

12. Johns KJ, O'Day DM. Pharmacologic management of keratomycoses. Surv Ophthalmol. 1988; 33: 178-188.

13. Maharana PK, Sharma N, Nagpal R, Jhanji V, Das S, Vajpayee RB. Recent advances in diagnosis and management of Mycotic Keratitis. Indian J Ophthalmol. 2016; 64 (5): 346-357. Doi: $10.4103 / 0301-4738.185592$.

14. Savani DV, Perfect JR, Cobo LM, Durack DT. Penetration of new azole compounds into the eye and efficacy in experimental Candida endophthalmitis. Antimicrob Agents Chemother, 1987; 31: 6-10.

15. Foster CS. Miconazole therapy for keratomycosis. Am J Ophthalmol, 1981; 91: 622-629.

16. Shu TH, Hussein A, Kursiah MR. Conjunctiva necrosis following Subconjunctival Amphotericin B injection in fungal keratitis. Cureus, 2019; 11 (9): e5580.

17. El-Sayed SH, Wagdy FM, El-Hagaa AA, Mottawea EF. Topical Amphotericin B versus Subconjunctival Fluconazole injection in the management of fungal keratitis. Menoufia Med J. 2016; 29 (3): 601-605.

18. Shah SIA, Shah SA, Rai P, Abbasi SA, Fatima H, Soomroet AA. Etiology of infectious keratitis as seen at a tertiary care center in Larkana, Pakistan. Pak J Ophthalmol. 2016; 32 (1): 48-52.

19. Ausayakhun S, Chaidaroon W. Suppurative keratitis: clinical analysis of 224 cases. Thai J Ophthalmol. 1992; 6: $1-7$.

20. Wong KH, Kam KW, Chen LJ, Young AL. Corneal blindness and current major treatment concern-graft scarcity. Int J Ophthalmol. 2017; 10 (7): 1154-1162.

21. Yee RW, Cheng CJ, Meenakshi S, Ludden TM, Wallace JE, Rinaldi MG. Ocular penetration and pharmacokinetics of topical Fluconazole. Cornea, 1997; 16: $64-71$.

22. Behrens-Baumann W, Klinge B, Ruchel R. Topical Fluconazole for experimental Candida keratitis in rabbits. Br J Ophthalmol. 1990; 74: 40-42.

23. Yilmaz S, Maden A. Severe fungal keratitis treated with Subconjunctival Fluconazole. Am J Ophthalmol. 2005; 140: 454-458.

24. Dev S, Rajaraman R, Raghavan A. Severe fungal keratitis treated with Subconjunctival Fluconazole. Am J Ophthalmol. 2006; 141: 783-784. 
25. Isipradit S. Efficacy of Fluconazole subconjunctival injection as adjunctive therapy for severe recalcitrant fungal corneal ulcer. J Med Assoc Thai. 2008; 91 (3): 309-315.

\section{Authors' Designation and Contribution}

Ambreen Gul; Senior Registrar: Concepts, Design, Literature search, final review.

Fuad Ahmad Khan Niazi; Professor: Concepts, Design, final review.

Ali Raza; Professor: Concepts, Design, final review. 